\title{
La prévention des troubles musculo- squelettiques : quelques enjeux épistémologiques
}

Musculoskeletal disorders prevention: some epistemological questions

La prevención de los trastornos músculo-esqueléticos : algunos desafíos

epistemológicos

\section{Fabien Coutarel, François Daniellou et Bernard Dugué}

\section{OpenEdition}

\section{Journals}

Édition électronique

URL : http://journals.openedition.org/activites/1550

DOI : $10.4000 /$ activites. 1550

ISSN : $1765-2723$

\section{Éditeur}

ARPACT - Association Recherches et Pratiques sur les ACTivités

\section{Référence électronique}

Fabien Coutarel, François Daniellou et Bernard Dugué, «La prévention des troubles musculosquelettiques : quelques enjeux épistémologiques », Activités [En ligne], 2-1 | avril 2005, mis en ligne le 02 avril 2005, consulté le 30 avril 2019. URL : http://journals.openedition.org/activites/1550 ; DOI : 10.4000/activites. 1550

\section{c.) (i) (9)}

Activités est mis à disposition selon les termes de la licence Creative Commons Attribution - Pas d'Utilisation Commerciale - Pas de Modification 4.0 International. 


\title{
La prévention des troubles musculo-squelettiques : quelques enjeux épistémologiques
}

\author{
Fabien Coutarel \\ François Daniellou \\ Bernard Dugué \\ Laboratoire d'Ergonomie des Systèmes Complexes \\ Université Victor Segalen Bordeaux 2, \\ Case 55, 146 rue Léo-Saignat, 33076 Bordeaux Cedex \\ Fabien.Coutarel@ergo.u-bordeaux2.fr
}

\begin{abstract}
Musculoskeletal disorders prevention : some epistemological questions.

Research concerning ergonomic practice has demonstrated its relevancy in several fields, especially concerning the prevention of Musculoskeletal Disorders (MSD). However, further knowledge in this field is necessary so as to reduce the exponential evolution of the number of affected workers. This paper aims, epistemologically, to discuss the criteria employed during scientific evaluations which are used by different research modes studying MSD : epidemiological, experimental and interventional research. The authors' position on this topic can be resumed as follows :

- each one of these three approaches is necessary for furthering MSD prevention ;

- stronger interactions are necessary between the different modes of research, however they are yet to be built ;

- criteria for scientific evaluation should take into consideration the different conditions intrinsic to the research activity characterizing each mode.
\end{abstract}

\section{KEYWORDS}

Epistemology, ergonomics research, intervention research, evaluation, prevention of musculoskeletal disorders

Malgré un développement mondial maintenant considérable des recherches sur les troubles musculo-squelettiques (TMS), la progression de la pathologie n'est pas stoppée. Pour ce qui est de la France, la forme de la courbe des reconnaissances continue à être exponentielle. Cet état de fait interroge sur la relation entre les connaissances permettant de comprendre l'ampleur de la pathologie et son étiologie, et les connaissances permettant de contribuer à des mesures de prévention.

Force est de constater, en effet, que la recherche sur les interventions de prévention tient une place extrêmement mineure dans la recherche internationale sur les TMS. Le congrès international PREMUS, organisé tous les trois ans sur le thème des troubles musculo-squelettiques, est une excellente vitrine des efforts de recherche dans le monde. En Juillet 2004, à Zurich, sur près de 300 communications, seulement une petite vingtaine présentaient une réflexion précise sur les conditions d'intervention en vue de la prévention des TMS. L'un des thèmes les plus représentés était celui de «l'identification des cas », c'est-à-dire la question médico-légale de la désignation nosographique et de la reconnaissance d'une pathologie en vue de sa réparation financière.

Nous voudrions ici avancer les propositions suivantes : 
- A côté de la recherche reconnue sur la contribution étiologique de différents facteurs de risques, il existe maintenant un fort courant proposant une approche de la relation entre activité et risque d'apparition de TMS, où la subjectivité joue un rôle intégrateur. C'est-à-dire que le rapport subjectif au travail jouerait un rôle important dans la relation entre les facteurs étiologiques identifiés et l'activité du travailleur.

- Cette approche conduit à proposer des formes d'intervention ergonomique distinctes de celles qui découlent de l'approche en termes d'exposition aux facteurs de risque.

- Cette approche est en difficulté pour faire les preuves de son intérêt scientifique, du fait des critères d'évaluation dominants dans le domaine de la santé.

- Pour autant, les recherches les mieux évaluées suivant les mêmes critères ne parviennent pas suffisamment à orienter des formes de prévention efficaces.

- Selon notre point de vue, les progrès de la prévention des TMS ne passent pas par l'élimination d'une de ces deux approches mais par l'organisation de la mise en tension entre les deux. Les progrès de la prévention des TMS passent probablement par l'organisation de confrontations hétérogènes entre des recherches relevant des critères épistémologiques du contrôle des facteurs, et des recherches relevant des critères épistémologiques propres à l'approche compréhensive de l'activité humaine.

Il est maintenant largement admis, à propos de nombreuses pathologies (infection VIH, addictions), que la connaissance de la contribution étiologique de différents facteurs à une pathologie ne suffit nullement à définir des actions de santé efficaces. Les acteurs n'ont pas pour seule activité de s'exposer aux facteurs de risques ou de les éviter, et les compromis qu'ils construisent mobilisent de nombreux autres critères que la prévention d'une pathologie. La recherche sur la prévention de la contamination par le virus VIH a largement montré que l'identification de l'agent pathogène et des modes de transmission, et l'information sur les mesures de prévention efficaces (préservatif) ne suffisaient pas à prévenir la propagation de la maladie. La compréhension des autres objectifs et contraintes des acteurs concernés, des déterminants de leurs conduites, est essentielle, et nécessite le recours aux Sciences Humaines (Moatti, \& Spire, 2003). Les travaux de Becquet et al. (2004), par exemple, sur la prévention de la contamination par le lait maternel en Afrique, ont mis en évidence l'indispensable association de médecins et d'anthropologues pour mettre en place des conditions susceptibles de convaincre les femmes contaminées de ne pas allaiter. Dans plusieurs domaines, la force des associations de patients a rendu incontournable la prise en compte de leurs points de vue, par exemple sur l'acceptabilité des traitements.

Curieusement, et à la différence de la contamination par le VIH, les troubles musculo-squelettiques ne semblent pas donner lieu à un développement de recherche en sociologie ou en anthropologie. Il semble que ce soit essentiellement au sein de l'ergonomie et de la psychologie du travail que, depuis la fin des années 1990, des travaux plus ou moins convergents proposent un faisceau d'approches distinct de celles en termes de facteurs de risques.

\section{1.- Deux visions en présence}

Il est impossible de faire ici une revue exhaustive des milliers de publications portant sur les TMS. Une première catégorisation simplificatrice permet cependant, pour poser les termes du débat, de distinguer deux familles de positions en présence.

- La première position pourrait se définir ainsi :

$\mathrm{P} 1$ : «Les TMS sont une atteinte de l'organisme résultant de l'exposition des sujets à une combinaison pathogène de facteurs biomécaniques et psychosociaux. Les recherches permettent de préciser la contribution de chacun de ces facteurs, en contrôlant les facteurs de confusion. L'intervention de 
prévention doit viser à supprimer ou au moins à diminuer les combinaisons pathogènes de ces facteurs ».

Cette position est, à quelques variantes près, celle qui est présente dans les grands manuels de référence (Putz-Anderson, 1988 ; Pujol, 1993 ; Kuorinka, \& Forcier, 1995 ; Bernard, 1997 ; Buckle, \& Devereux, 1999). Appelons M1 le monde scientifique qui se reconnaîtrait approximativement dans cette formulation P1.

- La deuxième position, moins stabilisée, peut cependant faire aussi l'objet d'une tentative de formulation :

P2 : «Le corps humain est indissociablement organisme biologique et subjectivité. L'être humain, pour construire sa santé, a besoin de pouvoir exercer une influence personnelle sur les milieux dans lesquels il agit. Certaines formes répétitives d'organisation du travail empêchent l'exercice de gestes porteurs de sens pour le travailleur, leurs mouvements étant largement déterminés par des contraintes mécaniques externes. L'hypersollicitation de l'organisme est aussi une hyposollicitation du corps habité par la subjectivité, une atrophie du pouvoir du sujet d'agir sur la situation à travers son corps. Dans ces conditions, l'intervention de prévention doit viser la restauration de marges de manœuvre, permettant le déploiement par le sujet de gestes professionnels, dont la richesse soit adaptée à la diversité des conditions de production. Ces marges de manœuvre sont à rechercher dès les processus de conception, elles concernent les dispositifs techniques et la structure organisationnelle, mais aussi l'acquisition par les individus et par les collectifs de nouvelles ressources pour faire face aux variations de production ».

On trouvera des positions voisines de cette formulation dans Bourgeois (1998), Daniellou (1998), Hubault (1998), Dejours (1999), Pezé (1999), Bourgeois, Lemarchand, Hubault, Brun, Polin, \& Faucheux (2000), Clot (2001), et une revue de questions sur le sujet dans Coutarel (2004). Soit M2 le monde scientifique qui se reconnaîtrait approximativement dans cette formulation P2.

Les deux formulations $\mathrm{P} 1$ et $\mathrm{P} 2$ ci-dessous relèvent de deux mondes scientifiques différents, et peuvent paraître irréconciliables. Le point de vue qui est défendu ici est que la prévention des TMS ne peut se passer :

- ni d'un approfondissement de chacune des positions P1 et P2, grâce à des confrontations internes à chacun des deux mondes M1 et M2 qui les portent, suivant les règles respectives de chacun d'entre eux ;

- ni d'une confrontation hétérogène entre ces deux mondes, dans le respect des différences épistémologiques entre l'un et l'autre.

Cette proposition renvoie à la question fondamentale de savoir si les règles définissant la science sont communes à toutes les disciplines et à tous les objets, ou si elles relèvent d'un travail interne à chaque discipline (Daniellou, 1996, p. 11) : «Certains [...] défendent l'unicité de la science, et le caractère achevé et stable des règles qui gouvernent la recherche scientifique. [...] D'autres, au contraire, insistent sur la diversité des objets des disciplines scientifiques. [...] Dans ces cas, les règles qui gouvernent l'accès à la dignité scientifique doivent être retravaillées à la lumière des spécificités des objets des disciplines, et la réflexion épistémologique est perçue non comme la référence à un code donné une fois pour toutes, mais comme une composante de l'activité d'élaboration de la communauté disciplinaire ». Nous adoptons ici la deuxième position.

Examinons maintenant quelques objections réciproques que peuvent se faire les mondes M1 et M2. 


\section{2.- Objections réciproques}

Malgré le caractère socialement dominant du monde M1 dans le champ de la santé au travail, et l'extrême qualité de nombreux travaux publiés suivant les critères de ce monde, la position P1 reste confrontée à certaines objections sérieuses.

\section{1.- Des limites à la quantification biomécanique}

Certaines de ces objections peuvent être émises de l'intérieur et avec le langage même du monde M1. Par exemple, les tentatives de quantifier par des mesures la sollicitation d'un muscle ou d'une articulation se heurtent aux obstacles et aux difficultés suivants :

- les mesures électrophysiologiques réalisées en laboratoire sur une tâche stabilisée donnent des résultats extrêmement instables. De nombreuses études en soulignent la très grande variabilité inter-individuelle et, dans une moindre mesure, la variabilité intra-individuelle (Westgaard, 2000 ; Mathiassen, Burdof, \& van den Beek, 2002 ; Nordlander et al., 2004), et nous invitent donc à une grande prudence concernant l'utilisation qui peut en être faite. L'une des explications de cet état de fait est la vicariance des utilisations des degrés de liberté du poignet, du coude et de l'épaule, par une même personne au cours du temps, et selon les personnes (Aublet-Cuvelier, Aptel, \& Weber, 2004);

- la quantification des angulations par enregistrement vidéo en situation de travail n'a aucun sens si elle porte sur le cycle standard, du fait de l'extrême variabilité des cycles. De plus, l'évaluation de la charge biomécanique par un échantillonnage d'images fixes est largement mise en cause, le nombre d'images par seconde ne devant, selon certains auteurs (Kumar \& al., 2004), pas descendre en dessous de 5 si l'on veut obtenir une estimation fiable.

- Aptel et Claudon (2004) nous rappellent que si l'électromyographie permet de faire un certain nombre de mesures, la quantification ainsi obtenue des facteurs de risque reste difficile à interpréter.

- Il n'existe aujourd'hui aucune mesure quantitative de l'exposition qui puisse facilement être liée à un risque en ce qui concerne les faibles niveaux d'exposition (Westgaard, 2000). L'hypothèse des «fibres de Cendrillon» (Aptel, \& Gaudez, 2003) suppose une activation constante d'un petit nombre de fibres musculaires pour expliquer l'apparition de pathologies dans des cas de faible exposition. Mais l'électromyographie de surface ne permet pas d'investir cette piste.

- Des facteurs de risque psychosociaux pourraient être associés avec de faibles niveaux d'activité musculaire. Mais cette association potentielle est difficile à détecter dans des données de terrain (Westgaard, 2000).

- Toujours en ce qui concerne les faibles niveaux d'exposition biomécanique et psychosociale, les caractéristiques individuelles des travailleurs deviennent prépondérantes pour expliquer la survenue de pathologies : "in the studies with low biomechanical and psychosocial exposures it has proved increasingly difficultto relate the individual variation in musculoskeletal health to workrelated risk factors by conventional statistical analyses. [...] Thiscan be a methodological problem ; the risk indicators may not be sufficiently pointers to the environmental condition with risk 
potential" (Westgaard, 2000, p. 572) ${ }^{1}$. De plus, la prépondérance des caractéristiques individuelles pour définir un niveau acceptable d'exposition dans les cas de faible exposition biomécanique est également susceptible de varier dans le temps pour un même travailleur.

Il existe certaines mesures d'expositions très clairement pertinentes, qu'il est important d'utiliser pour prévenir les TMS. Cependant, dans de nombreux autres cas, les outils disponibles ne sont pas efficaces : "we must deal with the clear risk exposures, but also assume that undetected risk factors exist. A successful intervention that improves biomechanical exposure by conventional criteria does not guarantee good health" (Westgaard, 2000, p. 573) ${ }^{2}$.

Compte tenu de ces difficultés, il apparaît :

- que de nombreuses évaluations biomécaniques quantitatives publiées sont contestables ;

- que l'évaluation quantitative des sollicitations biomécaniques sur l'ensemble des postes d'une situation industrielle quelconque est impossible.

Ces arguments ne mettent aucunement en cause :

- l'intérêt d'une analyse biomécanique qualitative, voire quantifiée, de certaines situations de travail particulières, pour comprendre par exemple les phases les plus sollicitantes ou éclaircir les raisons d'une apparition énigmatique de TMS ;

- l'intérêt d'approches quantitatives dans des conditions contrôlées pour comparer, par exemple, les avantages et inconvénients de différentes variantes d'un même outil (Roquelaure, 1999).

L'analyse quantitative de manière générale présente de nombreux intérêts pour la recherche, et ces intérêts sont à juste titre vigoureusement défendus dans la littérature (Lasfargues, Rauquelaure, Fouquet, \& Leclerc, 2003 ; Claudon, \& Aptel, 2004). Cependant la pertinence du type de mesures auxquelles elle conduit pour la recherche de terrain mérite systématiquement d'être évaluée, au regard des difficultés précisées ci-dessus : " on ne peut pas considérer "le quantitatif en santé au travail" comme un programme de recherche en soi. Au contraire, c'est la démarche ergonomique qui pose à un moment donné, sur un sujet et dans un contexte donnés, la question d'une intégration de matériaux statistiques dans sa méthodologie » (Volkoff, 2005). C'est également la position défendue par Lasfargues et al. (2003).

Nous l'avons vu, la mise en place de mesures quantitatives présente se heurte, dans certaines conditions, à des obstacles qui ne sont pas aujourd'hui surmontés. Mais, comme nous le rappelle Westgaard (2000, p. 573) : "the worker often has a good perception of critical exposure factors, and may identify tasks causing the most [mental or physical] stress"3. L'ergonomie francophone ne peut que se rassurer d'une telle affirmation qui rend au travailleur une part de son intelligence : « [...] le repérage des difficultés et leur explication par les travailleurs étaient en général une source d'information immense et sous-estimée. [...] Nous avons pu comprendre à quel point l'activité cognitive des travailleurs était considérable : ils avaient en tête des problèmes très complexes et des solutions qui ne l'étaient pas moins ; mais cette activité cognitive a d'abord besoin d'être reconnue et aussi d'être explorée selon des modalités propres » (Wisner, 1985, p. 25).

1. «Dans les travaux où ont été notés de faibles niveaux d'exposition aux facteurs biomécaniques et psychosociaux, il s'est avéré très difficile de relier les évolutions individuelles en termes de santé musculo-squelettique à des facteurs de risque professionnels, au moyen des analyses statistiques conventionnelles. [...] Il pourrait s'agir d'un problème méthodologique ; les indicateurs de risque pourraient ne pas être des indicateurs suffisamment précis des conditions environnementales dans lesquelles se jouent ces risques potentiels ».

2. «Il faut se préoccuper des expositions clairement identifiées, mais aussi assumer le fait que d'autres facteurs de risque non détectés existent. Une intervention réussie parce qu'elle réduit l'exposition biomécanique, au regard des critères conventionnels, ne garantit pas une bonne santé ».

3. «Le travailleur a souvent une bonne perception des facteurs d'exposition critique, et peut identifier les tâches générant le plus grand stress (physique ou mental). » 
Il est difficile de suivre l'affirmation d'Aptel et Claudon $(2004$, p. 6$):$ « [... du point de vue de la recherche, il est évident que la quantification est la seule approche cohérente, informative et rationnelle pour évaluer les facteurs de risque et ainsi avancer rationnellement vers la maîtrise du risque. Il faut donc admettre que le mesurage est un impératif car il n'y a pas d'évaluation sérieuse sans mesurage rationnel des phénomènes ». Le mesurage nécessaire ne signifie pas que les facteurs de risque peuvent simplement et seulement se laisser cerner par la mesure quantitative. Nous sommes davantage enclins à suivre cet autre type de formulation : «La répétitivité apparaît comme un facteur de risque professionnel de SCC plus important que la force. [...] Par ailleurs, cette relation pose le problème de l'intérêt et de la fiabilité des évaluations strictement qualitatives dans la mesure où la force et la répétition sont aussi les facteurs de risque les plus difficiles à évaluer par ses méthodes » (Aptel, \& Cail, 2001, p. 58).

\section{2.- Des difficultés de la nosologie des TMS}

Le même type de discussions peut être proposé à propos de l'identification des cas, et donc de leur quantification. Il apparait en effet maintenant que les TMS peuvent être décrits comme un continuum entre des symptômes légers et une pathologie gravement invalidante, mais que ces signes ne suivent pas une ligne d'aggravation constante; il y a plutôt alternance de phases d'aggravation et de phases d'amélioration. Dans nombre de cas, plusieurs articulations peuvent être concernées, une amélioration des signes sur l'une d'entre elles pouvant s'accompagner d'une dégradation sur une autre (Aublet-Cuvelier et al., 2004). La profusion de recherches médico-légales sur l'identification des cas manifeste cette impossibilité, pour l'expert physiopathologiste, de dire nettement au juge ou aux partenaires sociaux : "c'est à partir de maintenant qu'il y a indiscutablement un syndrome du canal carpien ». Le médecin, lui, effectuera son diagnostic dans le cadre des tableaux sanctionnant un accord social sur la définition du cas.

Le monde M1 ne peut donc considérer que ses critères épistémologiques permettent de produire toutes les connaissances dont la prévention des TMS aurait besoin. Ceci est d'autant plus vrai que certains chercheurs du monde M1 soulignent les limites et les biais méthodologiques de certaines études épidémiologiques construites à partir des critères de cette approche (Hegmann, Thiese, \& Garg, 2004). Mais le monde M2 doit également faire face à des objections.

\section{3.- Des groupes-contrôle}

Le monde M1 adresse, de fait, au monde M2 une mise en demeure légitime de rendre compte de la scientificité de ses productions. L'une des formes de cette injonction est l'exigence, pour prouver la validité d'une forme d'intervention, de mettre en place un groupe-contrôle qui ne bénéficierait pas des effets de l'intervention (Westgaard, \& Winkel, 1997). Cette forme de validation, qui assure la qualité de la grande majorité des travaux en épidémiologie ou en pharmacologie, pose cependant une série de problèmes majeurs aux actions de prévention des TMS.

La première objection relève de la faisabilité sociale. Une entreprise sollicite une intervention ergonomique pour que la reconception d'une ligne de production intègre la prévention des TMS : on voit mal l'ergonome recommander une demi-ligne «améliorée » et une demi-ligne «témoin », pour le seul plaisir de renforcer ses arguments vis-à-vis de l'épidémiologie. Quant aux études où les « témoins » se trouvent dans d'autres ateliers ou entreprises que les « cas », on peut mettre en cause très sérieusement leur intérêt quand on voit le niveau d'ajustement aux spécificités de chaque situation que nécessite toute action de prévention des TMS.

Mais la deuxième objection est beaucoup plus fondamentale. Les praticiens de la prévention des TMS connaissent ce qu'ils appellent un « effet paradoxal » de leur intervention. Il est fréquent que l'intervention dans un atelier provoque une augmentation des plaintes dans un atelier voisin, où les 
ergonomes ne sont absolument pas intervenus. Cela est à mettre en relation avec le fait que toute action d'amélioration élève la norme sociale de référence, et que cette augmentation de la norme sociale dégrade mécaniquement la situation de tous ceux qui ne bénéficient pas de l'amélioration. Les sujets qui sont dans un groupe-témoin (sans intervention) ne sont donc pas en situation neutre «d'absence d'intervention». Ils sont en situation de «privation d'intervention », et voient leur situation se dégrader. On peut ainsi expliquer de nombreux résultats des études épidémiologiques, qui relèvent une dégradation, non systématique mais cependant récurrente, de l'état de santé des individus des groupes témoin. L'exposé et les figures présentés par Theorell (2004) à Zurich en est un exemple. Lorsque cette évolution négative est interprétée comme un reflet de la tendance historique de la population d'une entreprise, les résultats positifs sur la santé des travailleurs concernés par l'intervention sont alors surestimés. En effet, sans l'intervention, l'état de santé des travailleurs des ateliers voisins ne se serait pas nécessairement aggravé. Il faut donc reconnaître le biais que représente la dégradation des conditions de travail du groupe-contrôle pour l'évaluation de l'amplitude des effets positifs de l'intervention.

On se trouve donc dans une situation où :

- soit les «témoins » ne savent pas qu'ils appartiennent à un groupe contrôle ; cela implique un éloignement géographique important du lieu d'intervention qui rend suspecte toute comparaison entre les deux populations ;

- soit les « témoins » connaissent leur statut, et ils voient leur situation dégradée.

Pour pallier cette difficulté liée à l'absence d'intervention dans le groupe contrôle, et pour se prémunir de l'effet Hawthorne ${ }^{4}$, Westgaard et Winkel (1997) suggèrent l'usage d'un placebo : les membres du groupe-contrôle doivent recevoir « des solutions qui ont l'air bonnes mais qui n'apportent pas d'amélioration réelle sur le plan mécanique ». Sans même évoquer les questions éthiques que cette méthode soulève, on voit mal comment l'utiliser pour mettre à l'épreuve une méthode d'intervention qui prône une conception participative des postes de travail. Pourtant, l'implication de l'ensemble de l'entreprise dans la démarche est aujourd'hui reconnue comme une condition de la prévention (Buckle, \& Devereux, 2002 ; Vézina, \& Stock, 2005).

Tout cela ne remet pas en cause l'intérêt d'une discussion étroite entre l'ergonomie et l'épidémiologie (Coutarel, 2004), ou les méthodes statistiques de manière plus générale (Volkoff, 2005). Vézina et Stock (2005), qui ont également noté l'impossibilité de mettre en place un groupe-contrôle en situation d'intervention, nous le montrent. La construction de leur binôme ergonome/épidémiologiste a permis de dépasser les divergences méthodologiques pour construire une réelle collaboration et un certain nombre de compromis, dont les auteures révèlent tous les enjeux, toutes les difficultés, et, finalement, tous les bénéfices. Parmi ces compromis, nous pouvons repérer l'abandon de l'idée de groupe-contrôle externe à l'intervention pourtant voulu par l'épidémiologiste, et le choix d'un contrôle interne : comparaison des travailleurs exposés aux changements avant et après intervention et analyses multivariées pour expliquer les facteurs associés aux différences observées. Il est également important de noter que si l'intervention ergonomique a concerné 23 travailleuses de 3 modules différents, un questionnaire avant / après a pu être proposé à l'ensemble des travailleurs de l'entreprise concernés par la production de jour $(\mathrm{n}=192)$, permettant de noter des évolutions plus générales de la population. Les résultats des entretiens réalisés par l'ergonome auprès des 23 travailleuses ont permis de donner du sens aux résultats statistiques obtenus, et notamment d'éviter des erreurs d'interprétation. Le pertinence de certaines questions standardisées pour l'entreprise considérée a également pu être revue. Ce travail témoigne de la richesse de ce type de collaboration entre ergo-

4. A la suite des travaux d'Elton Mayo, on appelle effet Hawthorne les résultats, positifs ou négatifs, qui ne sont pas dus aux facteurs mis en jeu par les travaux de recherche, mais aux effets que la conscience de participer à une recherche et d'être l'objet d'une attention spéciale exerce sur les travailleurs. 
nome et épidémiologiste, dont on peut remarquer qu'elle ne passe pas nécessairement par la mise en place de groupe-contrôle externe. Cette collaboration nécessite cependant un certain nombre de conditions : un investissement important et de réelles négociations entre ergonome et épidémiologiste pour dessiner les compromis les plus pertinents du point de vue des enjeux différents de chacun des chercheurs, et une construction sociale lourde du projet de recherche qui implique l'ensemble de l'entreprise et de ses salariés dans la démarche. L'effectif de travailleurs concerné par la recherche est sans doute également un élément important de ce type de démarche même si les auteures ne mentionnent pas cet aspect.

\section{3.- Mettre en rapport la nature des propositions et les formes de preuves}

L'absence actuelle de confrontation organisée entre les mondes M1 et M2 se traduit par la situation paradoxale suivante. Des méthodes d'intervention susceptibles d'une plus grande efficacité dans le domaine de la prévention des TMS ont été élaborées et formalisées, en particulier depuis 1998, et font l'objet d'un certain consensus au sein d'une communauté limitée de chercheurs et de praticiens en ergonomie. Ces modèles, cependant, ne parviennent pas à se faire reconnaître dans les forums internationaux de recherche sur les TMS, dont les critères épistémologiques relèvent du monde M1. Les formes d'administration de la «preuve » admises par le monde M1 ne semblent pas compatibles avec les propositions que veut défendre le monde $\mathrm{M} 2^{5}$.

Nous proposons ici l'approche suivante :

- formuler l'état actuel de ces propositions méthodologiques avancées par M2 ;

- ouvrir la discussion sur les mécanismes de validation déjà mis en œuvre au sein du monde M2 pour affiner ces modèles ;

- proposer des formes de confrontation entre M1 et M2 à propos de ces modèles.

\section{1.- L'évolution des modèles d'intervention}

L'histoire de la réflexion sur les interventions de prévention des TMS au sein du monde ergonomique francophone est présentée par Coutarel (2004). Nous en retiendrons les éléments suivants. Les tentatives d'intervention basées sur la seule diminution des facteurs pathogènes ont donné des résultats décevants, ou des résultats inattendus. Pour faire face à cette difficulté, un réseau de praticiens et de chercheurs français, coordonné par l'Agence Nationale pour l'Amélioration des Conditions de Travail (ANACT), a tenté de développer l'organisation d'une pratique réflexive sur les interventions. Ce travail a donné lieu à un séminaire (Bourgeois, 1998), où de nouvelles propositions de formulation de l'étiologie des TMS sont apparues. D'autres réflexions du même type ont eu lieu à l'initiative de la Mutualité Sociale Agricole (INRS, MSA, \& CNAM, 2000). La parution du livre de Bourgeois et al. (2000) a formalisé une étape supplémentaire, intégrant une réflexion sur le geste professionnel $^{6}$. Les interventions se référant à ce modèle se sont multipliées, et en 2003 le constat généralisé était le suivant : les interventions se référant à ce modèle ont une efficacité avérée, mais celle-ci semble de relativement courte durée. Un nouveau séminaire de l'ANACT en juillet 2004 sur

5. Le même type de paradoxe se trouvait par exemple dans une situation médiévale bien connue. Lorsqu'une femme affirmait l'impuissance de son mari - seul argument susceptible de faire annuler le mariage -, l'intéressé devait faire la preuve de ses capacités, au cours de travaux pratiques surveillés par deux ecclésiastiques. Il n'est pas certain que la forme de preuve fût là non plus compatible avec ce qui était à démontrer.

6. Le travail de Fernandez (2004) constitue aujourd'hui un bel exemple de recherche dans cette voie. 
la «prévention durable » a permis d'analyser les raisons de cette situation et d'en tirer de nouvelles leçons, sur lesquelles nous reviendrons.

La méthodologie générale d'évolution des modèles d'intervention est donc la combinaison, d'une part, d'une pratique réflexive et collective des praticiens, et, d'autre part, de la contribution de la « recherche sur la pratique », mise en place par des équipes de recherche (Daniellou, 1996, 1999; Coutarel, 2004). Le mouvement de ce type de recherche est le suivant. Au début d'une intervention, l'intervenant-chercheur s'astreint à expliciter le modèle qui va guider son intervention, et les résultats qu'il en attend. Il met en place le recueil de données permettant d'évaluer les résultats effectifs. L'écart entre les résultats effectifs et les résultats attendus sert de base à une évolution du modèle, qui sera à nouveau mis à l'épreuve dans d'autres interventions.

\section{2.- L'état actuel des propositions sur l'intervention de prévention des TMS}

Le séminaire «La prévention durable des TMS » mis en place par l'ANACT en Juillet $2004^{7}$ a fait apparaître un certain consensus sur les conditions de réussite d'une intervention de prévention, que l'on pourrait résumer comme suit.

- L'approche de la prévention des atteintes à la santé que constituent les TMS doit être articulée avec une réflexion sur les conditions de l'efficacité de l'entreprise et du processus productif considéré. Les difficultés rencontrées par les salariés dans la réalisation de leur tâche, qui donnent lieu aux TMS, se manifestent aussi par des problèmes de production ou de qualité dont le traitement doit être combiné avec la prévention des pathologies.

- L'action de prévention doit être conduite comme un projet, avec une implication de la direction de l'entreprise, un responsable clairement identifié, et un processus participatif qui associe les opérateurs concernés et l'encadrement de proximité. L'action de prévention doit aussi s'insérer dans les projets de l'entreprise, en particulier dans ceux qui assurent son développement ; elle doit aussi se positionner vis-à-vis des autres projets de prévention, en particulier ceux relatifs à l'évaluation des risques.

- L'approche par le travail permet de sortir de la segmentation du fonctionnement de l'entreprise en différents « domaines » (hygiène-sécurité, qualité, productivité, marketing, ...). Les différentes logiques correspondantes sont prises en compte comme autant de contributions à la lecture des déterminants des activités de production.

- Il est souhaitable d'agir simultanément sur différents domaines : conception des produits, procédés de production, dispositifs techniques, organisation du travail, accueil des nouveaux, formation, etc.

- Le processus est basé sur une compréhension de l'activité dans des situations existantes, et sur des simulations des formes possibles de l'activité future.

- Un enjeu important est d'obtenir rapidement des améliorations même limitées, pour permettre aux opérateurs et à l'encadrement de sortir de la conviction qu' "il n'y a pas moyen de faire autrement $\gg($ Daniellou, 1998 ; Nahon, \& Arnaud, 1999). En effet, tant que ce type de défense est en place chez les différents acteurs, toutes les démonstrations de la gravité du phénomène TMS se heurtent à cette attitude fataliste.

7. Les actes de ce séminaire sont actuellement en cours d'élaboration. Ils seront prochainement disponibles sur http:// www.anact.fr 
- Pour être efficace, l'action de prévention doit concerner non seulement le champ des changements techniques et organisationnels, mais aussi celui de la mobilisation des acteurs et de leurs relations, ainsi que celui des représentations dominantes sur la santé et sur les liens efficacité/ santé.

A l'issue de premières actions présentant ces caractéristiques, il est fréquent de constater des changements notables, une amélioration de la situation sanitaire et de la qualité, ainsi qu'une transformation de l'expression des salariés et de l'encadrement. Cependant, ces améliorations sont généralement mises à mal au bout de quelques années. L'interprétation qui peut être faite de cette dégradation, au sein du modèle étiologique proposé ici, est la suivante. Durant un projet qui présente les caractéristiques qui viennent d'être décrites, des conditions très inhabituelles sont réunies dans l'entreprise : approche multilogique et multiniveau (de la conception fine des postes de travail à la stratégie de l'entreprise) ; passage des opérateurs d'un statut de plaignants à celui d'acteurs ; prise en compte des contraintes de l'encadrement ; coopération des différents acteurs ; négociation du cahier des charges avec les fournisseurs ; réflexions sur l'organisation, les rotations. Tout cela tient grâce à la forme de présence (Hubault, 2004) qu'incarne l'animateur, en général intervenant extérieur, de sorte que se construit ce que l'on pourrait appeler une « démocratie industrielle provisoire » (Daniellou, 2004). La réalité des projets montre qu'après le départ des intervenants, cette manière de fonctionner disparait progressivement, parce qu'aucune instance de l'entreprise ne porte plus l'ensemble de ces conditions.

Le séminaire ANACT 2004 a permis de formuler des conditions d'une prévention durable des TMS, qui peuvent être résumées comme suit. La prévention durable des TMS ne relève pas du succès définitif, de l'éradication, comme cela a été le cas pour quelques maladies infectieuses. Ce qui est en jeu, c'est qu'il y ait, pour mener une action soutenue face aux TMS, des structures durables (internes ou externes à l'entreprise), qui assurent la forme de présence qui vient d'être décrite, qui assument les conflits de logique entre productivité et prévention, dans un environnement toujours changeant, et qui aient des comptes à rendre sur le fait que les TMS soient maintenus à un niveau « aussi bas que possible» (suivant le concept ALARA : "as low as reasonably achievable"). Cette liste de conditions peut sembler inatteignable. Or, ce mode de gestion des risques est couramment utilisé et largement maîtrisé dans d'autres domaines : la gestion du risque lié à des rayonnements ionisants dans les installations nucléaires de base, par exemple, possède toutes les propriétés ci-dessus.

\section{3.- Quelles formes de l'évaluation pour l'intervention ergonomique ?}

Nous avons jusque-là tenté de montrer que les critères d'évaluation des travaux menés dans le monde épistémologique M2 ne pouvaient être ceux appliqués par le monde M1 à ses propres travaux. Nous ne prétendons pas pour autant, évidemment, que les propositions du monde M1 devraient être dispensées des mises à l'épreuve (Stengers, 1993) qui permettent de les revendiquer comme scientifiques. Nous soulevons ici la question suivante : si jamais les propositions rassemblées dans le paragraphe 3.2 étaient valides, elles constitueraient une avancée notable par rapport au consensus international en matière de prévention des TMS et seraient susceptibles d'améliorer la prévention dans le monde. Mais leur pertinence est largement indécidable à l'intérieur du système de critères du monde M1. Il est au moins possible d'engager une discussion sur la question suivante : «quelles épreuves peuvent être mises en place pour tester la validité de ces propositions, épreuves compatibles avec la nature des propositions à démontrer?».

Nous en venons maintenant à proposer un certain nombre de types de données et de mises à l'épreuve, qui tiennent compte des particularités du monde M2 et de la nature des propositions à argumenter. Ces critères n'ambitionnent aucunement l'exhaustivité. Ils visent à prendre part à un débat épistémologique que la recherche de terrain doit continuellement conduire, nourrir, revendiquer et défendre. 
- Compte tenu de la complexité des facteurs en jeu dans l'apparition ou la diminution des TMS, et en contrepoint des grandes études épidémiologiques, des études monographiques sont nécessaires, qui portent sur des actions de prévention dont le contexte et les méthodes sont décrits précisément, même si les effectifs des populations concernées sont limités.

- Il est également souhaitable que les publications portant sur des interventions décrivent de manière plus explicite les différents modèles mobilisés : modèle de l'intervention, modèle de la pathologie, et modèle de l'opérateur et de son activité (Coutarel, 2003), ainsi que les éventuels écarts par rapport aux canons de la profession.

- L'évaluation d'une action de prévention doit combiner des données quantitatives, et des analyses compréhensives permettant d'accéder au vécu des acteurs par rapport à cette action. Des données uniquement quantitatives, interprétées par le seul chercheur, se plaçant en position surplombante, ne suffisent pas pour éclairer certains processus de changement.

- Il est nécessaire de prendre en compte le point de vue d'une diversité d'acteurs (travailleurs exposés au risque, encadrement de proximité, encadrement supérieur, représentants du personnel, professionnels de la prévention, fournisseurs,...).

- Il est utile de croiser différentes sources de données (Coutarel, 2004 ; Falardeau, \& Vézina, 2004), portant sur les pathologies, la production, la qualité, l'organisation du travail, les relations sociales, le vécu des travailleurs, etc. Cette confrontation de données hétérogènes présente deux avantages considérables: la compensation des limites intrinsèques de chacun des différents outils mobilisés, et une interprétation plus pertinente des résultats obtenus par chacun des outils. Nous avons précédemment rappelé les difficultés liées à l'interprétation des mesures par électromyographie, on trouvera également dans Molinié et Laville (2005) et Prunier-Poulmaire et Gadbois (2005) d'intéressantes précisions concernant l'utilité et les difficultés liées à l'utilisation de questionnaires.

- Concernant le moment de l'évaluation, il est en général souhaitable de ne pas raisonner seulement en comparaison avant/après, compte tenu de nombreux phénomènes plus ou moins cycliques connus à propos des TMS :

- La situation «avant» intervention devra être discutée, car il n'est pas indifférent qu'au moment où elle est réalisée, les travailleurs aient ou non déjà connaissance d'une volonté d'action vis-à-vis des TMS, du recours à des intervenants externes, etc.

- Il sera nécessaire de distinguer des phases de fonctionnement avec des moyens provisoires de celle du démarrage avec les moyens et l'organisation finaux.

- Les moments de l'évaluation doivent tenir compte de l'état d'avancement des processus de formation.

- Quelles que soient les améliorations apportées par le projet, il est classique que le démarrage soit un moment difficile, les travailleurs devant apprendre de nouveaux modes opératoires. L'évaluation au démarrage, indispensable pour corriger rapidement certaines difficultés rencontrées, doit être complétée par une évaluation plusieurs mois plus tard, qui permettra mieux de tirer le bilan du projet.

- De nouvelles évaluations, conduites plusieurs semestres plus tard, font apparaitre non pas principalement les effets du projet, mais ceux de l'évolution du contexte commercial, organisationnel, technique, social.

- A chaque phase d'évaluation, il importe de distinguer l'opinion des acteurs qui étaient déjà présents à la phase précédente, et celle des personnes qui ont été recrutées entre les deux.

- Dans le cas d'un questionnaire «trop simple », l'hypothèse que les « sujets » se souviennent de leurs réponses antérieures doit être prise en compte. 
- Concernant la prévalence des pathologies, et compte tenu des difficultés présentées plus haut, il est utile de multiplier les indicateurs :

- constats cliniques effectués par le médecin du travail ;

- questions portant sur la localisation et l'intensité des douleurs. Cependant, au-delà d'une semaine, les réponses sur l'intensité des douleurs («durant les 6 derniers mois ») ne sont pas fiables ;

- consultations médicales spontanées ou visites à l'infirmerie (fréquence, motif) ;

- questions sur la consommation d'antalgiques ;

- absentéisme

$-\ldots$

Le traitement de ces données est de nature à éclairer l'évolution des pathologies et de la production, entre différents moments. La question de l'imputabilité de ces évolutions demeure : dans quelle mesure les changements constatés sont-ils dus à l'intervention elle-même ? Ou bien résultent-ils d'autres évènements, de variations du contexte ? Plus précisément, dans quelle mesure sont-ils dus au fait qu'il y ait eu une intervention, à la nature des méthodes mises en œuvre, à la nature des transformations techniques mises en place, des changements d'organisation, des changements de style d'encadrement? Si l'énoncé P2 est valide, il est vain de chercher à quantifier la "part de la variance » qu'expliquerait chacun de ces facteurs. La nature des résultats obtenus est indissociable de la construction participative de la démarche. En revanche, les entretiens avec les acteurs permettent de comprendre comment ces différents ingrédients se sont assemblés dans la façon dont ils ont vécu la démarche et ses effets.

\section{4.- Organiser la confrontation des mondes}

La question de l'organisation de la confrontation entre les mondes M1 et M2 n'est pas une question spécifique de la prévention des TMS.

«Les discussions épistémologiques passent souvent rapidement sur le fait que l'évaluation des modèles scientifiques comporte deux aspects, qui ne se gèrent pas de la même façon :

- l'évaluation des qualités du modèle à l'intérieur de son espace de réduction;

- l'évaluation des qualités du modèle pour rendre compte du monde réel.

La première partie de l'évaluation des modèles se joue à propos de mondes particuliers, par exemple le laboratoire ${ }^{8}$, dont la complexité est artificiellement contrôlée.Le laboratoire n'est pas le monde, mais la production d'un monde particulier, où les phénomènes que décrivent les modèles sont épurés de dimensions perturbatrices [...]. Le scientifique au laboratoire peut créer un monde sans frottement, et montrer la prééminence de son modèle sur d'autres, dans un monde sans frottement.

Cette démarche réductrice, nécessaire à la production de modèles scientifiques, ne concerne pas le seul laboratoire. Sur le "terrain", elle conduira à prendre en compte certains paramètres, qui sont décrits par le modèle, et à considérer comme "de deuxième ordre" les éléments de la situation dont le modèle ne rend pas compte.

La recherche scientifique est largement structurée par les exigences de la validation "sous réduction “. Les institutions scientifiques organisent le contrôle du fait que le modèle rend correctement compte des conditions de réduction pour lesquelles il a été élaboré.

8. Ou l'enquête épidémiologique (note de 2004) 
Tout autre est la question de l'évaluation de la validité d'un modèle pour représenter non pas l'espace de réduction dans lequel il a été élaboré, mais le monde réel. Cette deuxième évaluation fait l'objet de beaucoup moins de débats dans la communauté scientifique »(Daniellou, 1997).

La nécessité de la confrontation entre des approches disciplinaires ne résulte pas des besoins de validation interne à chacun des mondes. C'est parce qu'il y a, dans le monde réel qui résiste à la modélisation exhaustive, des femmes et des hommes qui ont des TMS, des entreprises en difficulté, des préventeurs en action, que les scientifiques doivent confronter leurs modèles et leur pertinence pour l'action.

Ces confrontations nécessaires ne peuvent d'ailleurs pas être menées à bien entre les seuls scientifiques. Les acteurs des entreprises, les acteurs de la prévention, verront leur vie modifiée par les modèles de l'étiologie des TMS et de la prévention qui auront été retenus. S'ils n'ont pas à intervenir sur les processus de la validation interne à chaque discipline, leurs représentants ont quelque chose à dire sur les conséquences pour eux qu'aurait le fait de retenir, pour l'action, tel ou tel modèle nécessairement partiel.

Une confrontation, respectueuse et exigeante, des disciplines s'intéressant à la prévention des TMS est un enjeu incontournable des prochaines années. Elle passe par des projets de recherche où des chercheurs des deux mondes coopèrent « sur le terrain » (Vézina, 2001 ; Vézina, \& Stock, 2005). Elle suppose que les revues internationales se fassent l'écho et le vecteur de ces nécessaires controverses, et que les organisateurs de congrès veillent à donner toute leur place aux différentes positions épistémologiques. Enfin, cette confrontation nécessite aussi un débat sur les modèles de prévention des TMS à enseigner dans les écoles de management et les écoles d'ingénieur (Dull, 2004).

De nombreuses pesanteurs, propres à l'histoire et à la culture de chacun des mondes scientifiques, ne favorisent pas cette confrontation. Son développement dépendra, dans une large mesure, des exigences de la société vis-à-vis des TMS. Ce sont, dans bien des cas, les pressions des représentants des personnes concernées (syndicats ou associations de malades) et de l'Etat qui ont obligé les disciplines scientifiques à collaborer, les enjeux d'action urgente prenant le pas sur les enjeux internes de chaque discipline.

Il est probable que, dans le cas des TMS, on n'ait pas atteint une situation où le rejet massif de la pathologie par la société oblige les scientifiques à mettre en commun et en confrontation les ressources hétérogènes de différentes disciplines. Différents auteurs (Askenazy, 2004 ; Daniellou, 2004 ; Silverstein, 2004) insistent sur l'importance d'un renforcement de la condamnation sociale de l'ampleur des pathologies TMS.

Les chercheurs impliqués dans la compréhension de leur apparition et des conditions de leur prévention peuvent, sans attendre cet hypothétique mouvement, convenir que les exigences d'action appellent dès aujourd'hui d'inconfortables, mais sûrement fécondes, confrontations hétérogènes entre les modèles qu'ils produisent par des voies différentes.

Le présent article n'avait pas la prétention de produire une recension exhaustive des questions épistémologiques que soulève la recherche sur la prévention des TMS. Il vise à ouvrir un débat, qui mobilise une diversité de praticiens et de chercheurs également préoccupés par la réduction de cette pathologie. Le texte qui précède est écrit par des auteurs qui se reconnaissent plutôt dans la formulation « $\mathrm{P} 2 »$, mais qui souhaiteraient vivement avoir la chance d'approfondir cette réflexion et d'en rendre compte, conjointement avec d'autres auteurs porteurs d'autres points de vue.

\section{RÉFÉRENCES}

Aptel, M., \& Cail, F. (2001). Approche biomécanique des affections du tableau 57 des maladies professionnelles. In C. Hérisson, B. Fouquet, \& P. Codine (Eds.), Membre supérieur et pathologie 
professionnelle (pp. 53-61). Paris: Masson, Collection de pathologie locomotrice et de médecine orthopédique.

Aptel, M., \& Claudon, L. (2004). Physiologie musculaire et travail. In C. Hérisson, \& B. Fouquet (Eds.), Muscle et pathologies professionnelles (pp. 1-8). Paris: Masson, Collection de pathologie locomotrice et de médecine orthopédique.

Aptel, M,. \& Gaudez, C. (2003). Physiopathologie des TMS de l'épaule de type musculaire liés au travail : l'hypothèse des fibres de cendrillon. In C. Hérisson, \& B. Fouquet (Eds.), Ceinture scapulaire et pathologies professionnelles (pp. 1-12). Paris: Masson.

Askenazy, P. (2004). Les désordres du travail, Enquête sur le nouveau productivisme. Paris: Editions du Seuil et La République des Idées, 96 p.

Aublet-Cuvelier, A., Aptel, M., \& Weber, H. (2004). Fluctuation du statut clinique d'opérateurs exposés à des facteurs de risque de troubles musculosquelettiques du membre supérieur dans une entreprise d'assemblage d'équipement électro-ménager. In Actes du séminaire Anact (à paraître sur www.anact.fr), Les conditions d'une prévention durable des TMS, Paris, 7 \& 8 juillet 2004.

Becquet, R., Viho, I., Ekouevi, D.K., Sakarovitch, C., Goulheon, Z., Kouadio, S., Dabis, F., Bequet, L., Timite-Konan, M., \& Leroy, V. (2004). Uptake and determinants of exclusive breastfeeding with early cessation to prevent HIV-1 transmission through breastmilk. ANRS 1201/1202 Ditrame Plus project, Abidjan, Côte d'Ivoire. Poster $\mathrm{N}^{\circ}$ ThPeC7293. In Proceedings of The XV International AIDS conference, Bangkok, Thailand. July 2004.

Bernard, B. P. (Ed.). (1997). Musculoskeletal Disorders and Workplace Factors : a critical review of epidemiologic evidence for work-related musculoskeletal disorders of the neck, upper extremity, and low back. Cincinnati: DHNS (NIOSH) Publication n 97-141.

Bourgeois, F. (Ed.) (1998). TMS et évolution des conditions de travail. Les actes du séminaire, Paris 98. Editions ANACT, 133 p.

Bourgeois, F., Lemarchand, C., Hubault, F., Brun, C., Polin, A., \& Faucheux, J-M. (2000). Troubles musculosquelettiques et travail. Quand la santé interroge l'organisation. Paris: Editions ANACT, Collection outils et méthodes, $252 \mathrm{p}$.

Buckle, P., \& Devereux J. (1999). Work related neck and upper limb musculoskeletal disorders. European Agency for Safety and Health at Work.

Buckle, P., \& Devereux, J. (2002). The nature of work-related neck and upper limp musculoskeletal disorders. Applied Ergonomics, 33, 207-217.

Claudon, L., \& Aptel, M. (2004). Evaluation de l'activité musculaire et travail. Approche ergonomique, biomécanique et physiologique de la prévention des TMS. In C. Hérisson, \& B. Fouquet (Eds.), Muscle et pathologies professionnelles (pp.18-25). Paris: Masson, Collection de pathologie locomotrice et de médecine orthopédique.

Clot, Y. (2001). Psychopathologie du travail et clinique de l'activité. Education permanente, 146,.35-49.

Coutarel, F. (2003). La résistance du réel pour structurer les modélisations de la santé au travail : analyse du travail, activité et action sur le terrain : interactions entre modèles. Modèles et pratiques d'analyse du travail, 1988-2003, 15 ans d'évolution (Actes du XXXVIIIème congrès de la SELF, Paris, 2003) (pp. 451467). Toulouse: Editions Octarès.

Coutarel, F. (2004). La prévention des troubles musculo-squelettiques en conception : quelles marges de manoeuvre pour le déploiement de l'activité ? Thèse de doctorat en ergonomie, Université Victor Segalen Bordeaux 2, Editions du Laboratoire d'Ergonomie des Systèmes Complexes.

Daniellou, F. (Ed..) (1996). L'ergonomie en quête de ses principes. Toulouse: Octarès Editions.

Daniellou, F. (1997) Evolution de l'ergonomie francophone : théories, pratiques, et théories de la pratique. In M-F. Dessaigne, \& I. Gaillard (Eds.), Des évolutions en ergonomie (pp. 37-54). Toulouse: Octarès Editions, pp. 37-54.

Daniellou, F. (1998). Une contribution au nécessaire recensement des "repères pour affronter les TMS". In F. Bourgeois (Ed.), TMS et évolution des conditions de travail, Actes du séminaire Paris 1998, Etudes \& Documents (pp. 118-122). Lyon: Editions de l'ANACT. 
Daniellou, F. (1999). The ergonomist is a worker ? : that is the (epistemological) question. In N. Marmaras (Ed.), Proceedings of the Symposium : Strengths \& Weaknesses, Threats \& Opportunites of Ergonomics in Front of 2000 (pp. 21-28).

Daniellou, F. (2004, à paraître). Synthèses du séminaire. In Actes du séminaire Anact, Les conditions d'une prévention durable des TMS, Paris, 7 \& 8 juillet 2004.

Dejours, C. (1999). Incidences psychopathologiques des nouvelles formes d'organisation du travail, du management, et de gestion des entreprises. Archives des maladies professionnelles et de médecine du travail, $60(6), 534$.

Dull, J. (2004). How can interventions on work-related musculoskeletal disorders successfully be integrated into the business world? In Proceedings of the Fifth International Scientific Conference on Prevention of Work-related Musculoskeletal Disorders, Premus 2004, Vol. 1, pp. 55-56.

Falardeau, A., \& Vézina, N. (2004). Apport de différentes sources de données à la description comparée des contraintes et à l'amélioration d'un groupe de postes occupés en rotation. Pistes, 6 (1), 22 p. http:// www.pistes.uqam.ca/v6n1/articles/v6n1a7.htm

Fernandez, G. (2004). Développement d'un geste technique. Histoire du freinage en Gare du Nord. Thèse de doctorat de psychologie, CNAM, Laboratoire de psychologie du travail et de l'action, 201 p.

Hegmann, K., Thiese, M.S., \& Garg, A. (2004). Biases and Confounders in Musculoskeletal Disorders. In Proceedings of the Fifth International Scientific Conference on Prevention of Work-related Musculoskeletal Disorders, Premus 2004, Vol. 1, pp. 161-162.

Hubault, F. (1998). Articulations rigides pour coordinations souples ? Les TMS comme syndrome de la crise du modèle taylorien de régulation. In F. Bourgeois (Ed.), TMS et évolution des conditions de travail, Actes du séminaire Paris 1998, pp. 47-53.

Hubault, F. (2004, à paraître). Communication au séminaire sur la Prévention durable des TMS, organisé par l'Anact à Paris. In Actes du séminaire Anact, Les conditions d'une prévention durable des TMS, Paris, 7 \& 8 juillet 2004.

INRS, MSA, \& CNAM (2000). Actes du séminaire TMS 2000, Echanges sur les pratiques d'intervention, $83 \mathrm{p}$.

Kumar, S., Narayan, Y., \& Moro, L. (2004). Measurements and prediction of cumulative load in X-ray technologists. In Proceedings of the Fifth International Scientific Conference on Prevention of Workrelated Musculoskeletal Disorders, Premus 2004, Vol. II, pp. 377-378.

Kuorinka, I., \& Forcier, L. (Eds) (1995). Work related musculoskeletal disorders (WMSDs) : A reference book for prevention. London: Taylor \& Francis, $421 \mathrm{p}$.

Lasfargues, G., Roquelaure, Y., Fouquet, B., \& Leclerc, A. (2003). Pathologie d'hypersollicitation périarticulaire des membres supérieur. Paris: Masson, Collection Médecine du travail, $147 \mathrm{p}$.

Mathiassen, S.E., Burdof, A., \& Beek, A.J. van der. (2002). Statistical power and measurement allocation in ergonomic intervention studies assessing upper trapezius EMG amplitude. A case study of assembly work. Journal of Electromyography and Kinesiology, 12, 45-57.

Moatti, JP, \& Spire, B. (2003). Les enjeux économiques et sociaux de l'infection par le VIH a l'ère des multithérapies antirétrovirales : apports des sciences humaines et sociales. Médecine Sciences, 19(8-9), 878-884.

Molinié, A-F., \& Laville, A. (2005). Les surprises du longitudinal : les salariés répondent-ils n'importe quoi aux enquêtes portant sur leur travail actuel et passé. In S. Volkoff (Ed.), L'ergonomie et les chiffres de la santé au travail : ressources, tensions et pièges. Toulouse: Octarès Editions.

Nahon, P., \& Arnaud, S. (1999). Sortir de la boucle infernale... Essai de maîtrise des TMS dans trois abattoirs de porcs. In Actes du XXXIVème congrès de la SELF, 15-17 septembre 1999, Caen, pp. 147-153.

Nordlander, C., Balogh, I., Mathiassen, S.E., Ohlsson, K., Unge, J., Skerfving, S., \& Hansson, G.A. (2004). Precision of measurements of physical workload during standardised manual handling. Part I : Surface electromyography of $\mathrm{m}$. trapezius, $\mathrm{m}$. infraspinatus and the forearm extensors. Journal of Electromyography and Kinesiology, 14, 443-454. 
Pezé, M. (1999). Les athlètes du quotidien : approche psychosomatique et psychodynamique des TMS. In TMS et évolutions des conditions de travail - Les actes du séminaire, Paris 1998 (pp. 63-70). Lyon: Editions de l'ANACT, Etudes et Documents.

Prunier-Poulmaire, S., \& Gadbois, C. (2005). Quand le questionnaire s'impose à l'ergonome. In S. Volkoff Ed.), L'ergonomie et les chiffres de la santé au travail : ressources, tensions et pièges. Toulouse: Octarès Editions.

Pujol, M. (1993). Pathologie professionnelle d'hypersollicitation. Atteinte périarticulaire du membre supérieur. Paris: Masson, $168 \mathrm{p}$.

Putz-Anderson, V. (1988). Cumulative trauma disorders - A manual for musculoskeletal diseases of the upper limbs. London: Taylor \& Francis, 149 p.

Roquelaure, Y. (1999). Les activités avec instruments et préservation de la santé : approche interdisciplinaire. Thèse de doctorat d'ergonomie, Ecole Pratique des Hautes Etudes, 221 p.

Silverstein, B. (2004). Changing society's attitudes toward prevention of work-related musculoskeletal disorders. What can we learn from public health research ? In Proceedings of the Fifth International Scientific Conference on Prevention of Work-related Musculoskeletal Disorders, Premus 2004, Vol. 1, pp. 33-34.

Stengers, I. (1993). L'invention des sciences modernes. Paris: Editions La Découverte, 208 p.

Theorell, T. (2004). Work-related musculoskeletal disorder related to stress - how could they be prevented ? Changing individual behavior or developing organisation ? In Proceedings of the Fifth International Scientific Conference on Prevention of Work-related Musculoskeletal Disorders, Premus 2004, Vol. 1, pp. 45-46.

Vézina, N. (2001). La pratique de l'ergonomie face aux TMS : ouverture à l'interdisciplinarité. In Comptes rendus u congrès SELF-ACE 2001 "Les transformations du travail, enjeux pour l'ergonomie", vol. 1, pp. 44-60.

Vézina, N., \& Stock, S. (2005). Collaboration interdisciplinaire dans le cas d'une intervention ergonomique. In S. Volkoff (Ed.), L'ergonomie et les chiffres de la santé au travail : ressources, tensions et pièges, Octarès Editions, Toulouse.

Volkoff, S. (2005). L'ergonomie et les chiffres de la santé au travail : ressources, tensions et pièges. Toulouse: Octarès Editions.

Westgaard, R.H. (2000). Work-related musculoskeletal complaints : some ergonomics challenges upon the start of a new century. Applied Ergonomics, 31, 569-580.

Westgaard, R.H., \& Winkel, J. (1997). Ergonomic intervention research for improved musculoskeletal health : a critical review. International Journal of Industrial Ergonomics, 20, 463-500.

Wisner, A. (1985). Quand voyagent les usines. Paris: Syros, 195 p.

\section{RÉFÉRENCEMENT}

Coutarel, F., Daniellou F., \& Dugué, B. (2005). La prévention des troubles musculo-squelettiques : quelques enjeux épistémologiques. @ ctivités, 2 (1), 3-18, http://www.activites.org/v2n1/coutarel.pdf

\begin{abstract}
RESUMEN
La prevención de los trastornos músculo-esqueléticos : algunos desafíos epistemológicos.

La investigación acerca de la intervención ergonómica ya ha mostrado su interés en numerosos dominios, especialmente en el campo de la prevención de los Trastornos Musculo-Esqueléticos (TME). Sin embargo, resulta evidente que, para detener la evolución exponencial del número de trabajadores afectados, es necesario producir nuevos conocimientos.
\end{abstract}


El objetivo epistemológico de este artículo, es discutir los criterios de evaluación científica de los trabajos producidos por las distintas modalidades de investigación que abordan este problema : la investigación epidemiológica, la investigación experimental y la investigación sobre la intervención.

La posición defendida por los autores puede resumirse en lo siguiente:

- Cada una de estas modalidades de investigación resulta indispensable para mejorar la prevención ;

- En adelante, es necesaria una fuerte interacción entre las modalidades de investigación aunque, dicha interacción, deba todavía construirse;

- los criterios de la evaluación científica de los trabajos producidos deben tener en cuenta las diferentes condiciones de ejercicio de la actividad de investigación, típicas de cada modalidad.

PALABRAS CLAVE

Epistemología, investigación en ergonomía, investigación sobre la intervención, evaluación, prevención de Trastornos Musculo-Esqueléticos. .

\section{RÉSUMÉ}

La recherche sur l'intervention ergonomique a déjà révélé son intérêt dans de nombreux domaines, et plus particulièrement dans le champ de la prévention des Troubles Musculo-Squelettiques (TMS). Il reste évidemment de nombreuses connaissances à produire afin d'enrayer l'évolution exponentielle du nombre de travailleurs atteints par cette pathologie.

L'objectif épistémologique de cet article est de discuter des critères de l'évaluation scientifique des travaux réalisés par les différents modes de recherche qui investissent cette question : la recherche épidémiologique, la recherche expérimentale et la recherche sur l'intervention.

La position qui sera défendue par les auteurs peut être résumée comme suit :

- chacun de ces modes de recherche est indispensable à l'amélioration de la prévention ;

- une interaction plus forte entre ces modes de recherche est désormais nécessaire, mais reste à construire ;

- les critères de l'évaluation scientifique des travaux réalisés doivent tenir compte des conditions différentes d'exercice de l'activité de recherche, qui caractérisent ces modes.

\section{MOTS-CLÉS}

Epistémologie, recherche en ergonomie, recherche sur l'intervention, évaluation, prévention des Troubles Musculo-Squelettiques. 